\title{
Inflammatory cytokines in subarachnoid haemorrhage: association with abnormal blood flow velocities in basal cerebral arteries
}

\author{
K Fassbender, B Hodapp, S Rossol, T Bertsch, J Schmeck, S Schütt, M Fritzinger, \\ P Horn, P Vajkoczy, S Kreisel, J Brunner, P Schmiedek, M Hennerici
}

\begin{abstract}
Subarachnoidal release of inflammatory cytokines (interleukin (IL)-1ß, IL-6, and tumour necrosis factor (TNF)- $\alpha$ ) was characterised in 35 patients with subarachnoid haemorrhage (SAH) and control subjects and compared with development of complicating haemodynamic abnormalities in basal cerebral arteries and clinical outcome. Serial analysis allowed the observation of a subacute response profile of these key mediators of inflammation in the subarachnoidal space. This compartmentalised inflammatory host response was closely associated in time and extent with development of increased blood flow velocities in the basal cerebral vessels as recorded by transcranial Doppler sonography. Moreover, intrathecal secretion of inflammatory cytokines was significantly increased in patients with poor clinical outcome. Together, these findings suggest a role of excessive compartmentalised inflammatory host response in pathogenesis of cerebrovascular complications after SAH.

(F Neurol Neurosurg Psychiatry 2001;70:534-537)
\end{abstract}

Keywords: subarachnoid haemorrhage; inflammatory cytokines; cerebral blood flow; vasospasm; cerebral ischaemia

Subarachnoid haemorrhage (SAH) most commonly occurs when an aneurysm in a basal cerebral artery ruptures. Among patients who survive this event, the leading cause of death and disability is subsequent constriction of the large cerebral arteries causing delayed cerebral ischaemia, the "second stroke". ${ }^{2}$ Monitoring of cerebral blood flow velocities (CBFVs) in the large pial arteries identifies patients with $\mathrm{SAH}$ with raised risk for ischaemic complications. ${ }^{3-6}$ In $\mathrm{SAH}$, it has been shown in multiple studies that CBFVs on transcranial Doppler sonography are inversely related to vessel diameters on angiography. ${ }^{3-6}$ The noninvasive character of this technique enables serial investigations of developing haemodynamic abnormalities in an individual patient.
Earlier studies described inflammatory changes in SAH - that is, subarachnoidal and perivascular leucocytic infiltrates in the subarachnoidal space ${ }^{7-9}$ in relation to development of cerebral vasospasms. ${ }^{89}$ Moreover, the proinflammatory cytokines interleukin (IL) $-1 \beta$, IL-6, and tumour necrosis factor (TNF) $\alpha$ that orchestrate the cascade of inflammatory host response to infection and tissue damage ${ }^{1011}$ have been detected in the CSF of patients with SAH. ${ }^{12-16}$

These earlier findings suggest an inflammatory pathogenesis of vasospasms in SAH. In this study, we tested the hypothesis that the extent and pattern of secretion of key mediators of inflammation IL- $1 \beta$, IL- 6 , and TNF- $\alpha$ are associated with development of haemodynamic abnormalities in basal cerebral arteries and with clinical outcome.

\section{Patients and methods}

PATIENTS AND CONTROL SUBJECTS

Thirty five patients ( 21 women and 14 men), aged between 23 and 76 (median 56) years with SAH caused by aneurysmal rupture and presenting within 48 hours after onset of first symptoms were studied. Hunt and Hess scores to classify disease severity were 1 in $9 \%, 2$ in $11 \%, 3$ in $32 \%, 4$ in $29 \%$, and 5 in $18 \%$ of the patients. Extent and location of haemorrhage according to the Fisher scale were grade 2 in $12 \%, 3$ in $85 \%$, and 4 in $3 \%$. All except four patients underwent surgical obliteration of the aneurysm earlier than 72 hours after the onset of symptoms. Patients were mechanically ventilated when signs and symptoms of increased intracerebral pressure were present. Ventilation was necessary in $66 \%$ of the patients. Therapy consisted of combined induction of hypervolaemia, hypertension, and haemodilution (triple $\mathrm{H}$ therapy) alone in $49 \%$, combined with nimodipine in $20 \%$, with angiographically administered papaverin in $6 \%$, and with nimodipine alone in $6 \%$ of the patients. In all 35 patients, release of proinflammatory cytokines in CSF and peripheral blood and cerebral blood flow velocities in the basal cerebral arteries were assessed at day 5 . In addition, in 20 of the patients, all of these haemodynamic and biochemical variables were 
longitudinally investigated at days $1,2,3,5,7$, 9, and 11 after SAH.

Further, we included 20 control subjects (12 women and eight men), aged between 23 and 80 (median 40) years. These had lumbar puncture for initially suspected haemorrhage or meningitis, which was excluded by extensive clinical, neuroradiological, and laboratory investigation including CSF analysis.

MONITORING OF CBFVS AND ASSESSMENT OF CLINICAL OUTCOME

Abnormalities of CBFVs were monitored by non-invasive transcranial Doppler sonography using a DWL Multidop X TCD device (DWL Sipplingen, FRG). Systolic CBFV values were recorded transtemporally in the proximal segments of the anterior, middle, posterior, and basilar cerebral arteries as described before. ${ }^{17}$ All patients had follow up CT at least twice to assess possible delayed cerebral ischaemia. In each patient, the score of the Glasgow outcome scale (GOS) was determined. This study was approved by the ethics committee of the Mannheim Clinic, University of Heidelberg.

MEASUREMENT OF PROINFLAMMATORY

CYTOKINES

Plasma concentrations of proinflammatory cytokines (IL-1 $\beta$, IL- 6 , and $T N F-\alpha$ ) were
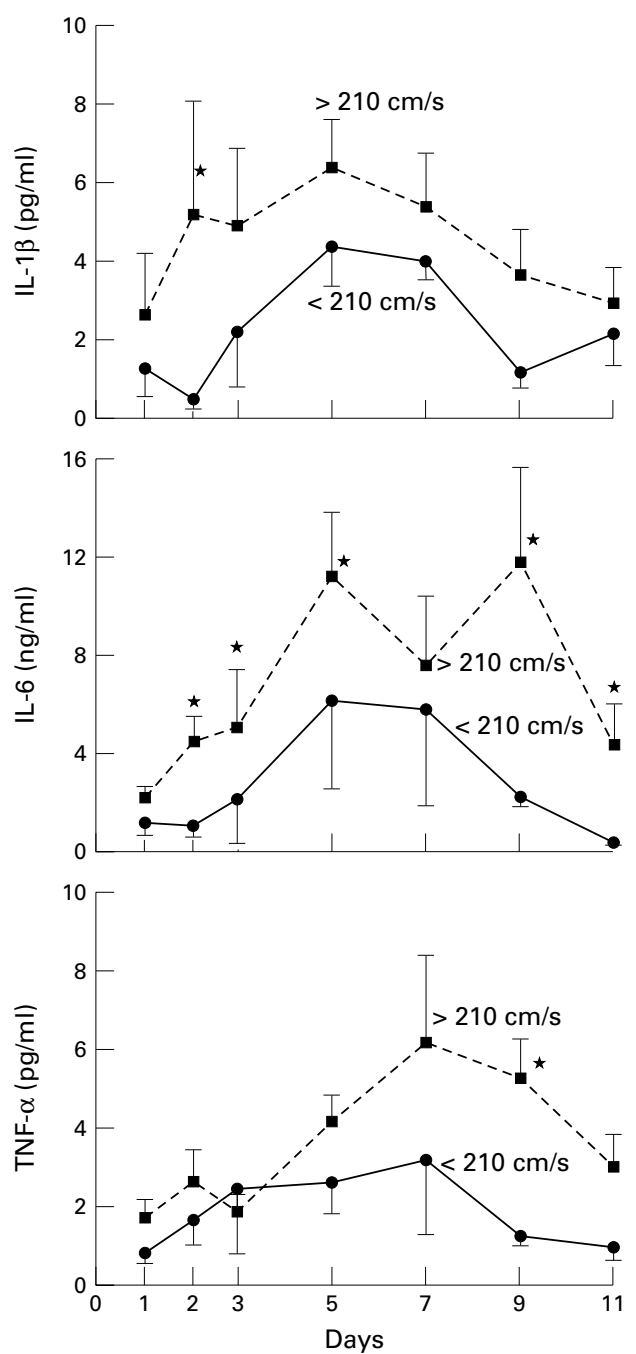

quantified with sandwich immunoassays ( $R$ and D Systems, Minneapolis, MN, USA) as described before. ${ }^{18}$ Briefly, a monoclonal antibody specific for the antigen of interest was coated onto a 96 well microtitre plate. In a single step reaction, samples were then incubated in the microtitre plate together with a second horseradish peroxidase linked monoclonal antibody specific for a different epitope. After washing, the bound enzyme-antibody conjugate was measured enzymatically with tetramethylbenzidine as the substrate. Adsorbence was measured at $450 \mathrm{~nm}$ on an MR 4100 spectrophotometer (Dynatech, Burlington, MA, USA) using $630 \mathrm{~nm}$ as the reference wavelength. The lower limits of detection for IL-1 $\beta$, IL- 6 , and TNF- $\alpha$ were $0.3 \mathrm{pg} / \mathrm{ml}, 0.3 \mathrm{pg} / \mathrm{ml}$, and $4.4 \mathrm{pg} / \mathrm{ml}$, respectively.

STATISTICAL ANALYSIS

Results are expressed as means (SEM). Statistical analysis involved a Mann-Whitney $U$ test and calculation of Spearman's correlation coefficient.

\section{Results}

CHARACTERISATION OF INTRATHECAL RELEASE OF PROINFLAMMATORY CYTOKINES IN SAH

Concentrations of IL- $1 \beta$, IL- 6 , and TNF- $\alpha$ in the CSF of control subjects were $0.00(0.00)$ $\mathrm{pg} / \mathrm{ml}, 0.0004(0.0001) \mathrm{ng} / \mathrm{ml}$, and $0.18(0.16)$ $\mathrm{pg}$, respectively. Compared with these normal values, CSF concentrations of proinflammatory cytokines in patients with SAH were massively increased in SAH (fig 1)-for example, CSF concentrations of IL- 6 were increased up to 10000 -fold. Longitudinal analysis showed a subacute response pattern for these cytokines, peaking between days 5 and 9 and declining thereafter (fig 1). In $\mathrm{SAH}$, concentrations of IL-1 $\beta$ and IL-6 were significantly lower in plasma than in CSF, showing the intrathecal origin of these mediators. Plasma and CSF concentrations of IL- $1 \beta$ and IL- 6 at day 5 were $2.21(0.70) v 5.78(0.83) \mathrm{pg} / \mathrm{ml}(\mathrm{p}<0.001)$ and $0.01(0.04) v 9.15(2.26) \mathrm{ng} / \mathrm{ml}(\mathrm{p}<0.0001)$, respectively. By contrast, serum and CSF concentrations of TNF- $\alpha$ did not differ significantly (3.72 (0.73) v $3.62(0.50) \quad \mathrm{pg} / \mathrm{ml}$; $\mathrm{p}=0.69)$. Samples of CSF obtained from extraventricular drainage (EVD) in $77 \%$ of the patients and by lumbar puncture in $23 \%$ did not differ in cytokine concentrations (data not shown).

\section{ALTERATIONS OF CBFVS AND ASSOCIATION WITH} SUBARACHNOID CYTOKINE SECRETION

Mean systolic CBFV subacutely increased during the study period of 11 days, peaking between days 7 and 11 when values reached a plateau (fig 2). Abnormalities were most pronounced in the middle cerebral arteries. Interestingly, changes in CBFVs paralleled those of CSF concentrations of inflammatory cytokines (figs 1 and 2). The response in the CSF, however, slightly preceded those of the haemodynamic abnormalities, peaking between days 5 and 9. We subdivided patients into two subpopulations with higher $(\geqslant 210$ $\mathrm{cm} / \mathrm{s}$, defined as the cut off value to diagnose 
Figure 2 Kinetics of changes in $C B F V$ (mean (SEM)) in intracranial basal arteries as determined by transcranial Doppler sonography $(n=20)$.

Reference values as derived from our earlier readings in 50 normal volunteers were 94.5 (1.9) $\mathrm{cm} / \mathrm{s}$ (middle cerebral artery, $M C A)$, $76.4(2.4) \mathrm{cm} / \mathrm{s}$ (anterior cerebral artery, $A C A), 53.2$ (1.6) $\mathrm{cm} / \mathrm{s}$ (posterior cerebral artery, $P C A)$, and 34.4 (1.7) cm/s (basilar artery, $B A){ }^{17}$
Figure 3 CSF concentrations (mean (SEM)) of IL-1 $\beta, T N F-a$, and IL-6 at day 5 in patients with better (GOS 3-5) or worse (GOS 1,2) clinical outcome of $S A H$. * $p<0.05$.

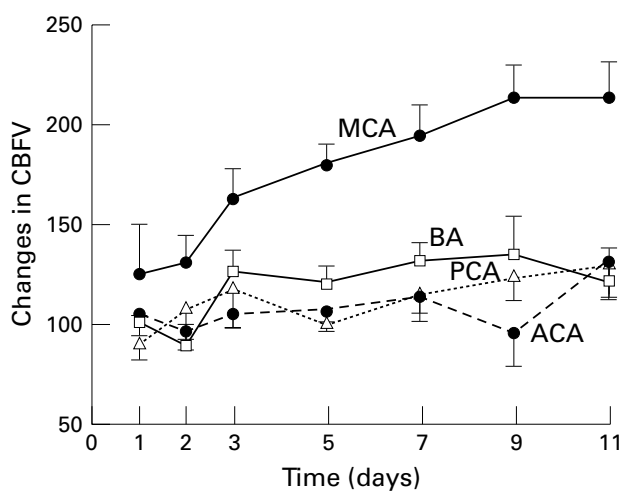

highly increased $\mathrm{CBF}$ cytokines ${ }^{17}$ ) or lower CBFVs $\quad(<210 \mathrm{~cm} / \mathrm{s})$. Development of CBFVs $\geqslant 210 \mathrm{~cm} / \mathrm{s}(\mathrm{n}=22)$ was associated with significantly increased CSF concentrations of IL-1 $\beta$, IL-6, and TNF- $\alpha$ compared with presence of $\mathrm{CBFV}<210 \mathrm{~cm} / \mathrm{s}(\mathrm{n}=13$, fig 1$)$.

CLINICAL OUTCOME AND ASSOCIATION WITH SUBARACHNOID CYTOKINE SECRETION

The GOS scores were 1 in $33 \%, 2$ in $27 \%, 3$ in $9 \%, 4$ in $6 \%$, and 5 in $24 \%$ of the patients. Concentrations of IL-6 at day 5 were significantly increased in patients with poor (GOS 1-2) compared with those with moderate or good (GOS 3-5) outcome, whereas concentrations of IL- $1 \beta$ and TNF- $\alpha$ exhibited a nonsignificant trend towards increased concentrations in the subpopulation with poor outcome (fig 3). Follow up CT showed signs of newly developed cerebral ischaemic injury in $46 \%$ of the patients with SAH. These patients exhibited a non-significant trend towards increased CSF concentrations of IL-6 at day 5 (12.11 (3.51) $\mathrm{ng} / \mathrm{ml} v 6.51(2.25) \mathrm{ng} / \mathrm{ml}, \mathrm{p}=0.08)$.

Concentrations of IL- $1 \beta(r=0.87, \mathrm{NS})$, IL-6 $(r=0.19, \mathrm{NS})$, and TNF- $\alpha(r=-0.16, \mathrm{NS}) \mathrm{did}$ not correlate with Hunt and Hess scores. Hunt and Hess scores were not significantly different in patients with higher or lower CBFVs (3.42 $(0.25)$ v 3.33 (0.36), $p=0.87)$. As expected, the scores, however, correlated with GOS $(r=-0.59, \mathrm{p}<0.005)$. Interestingly, GOS scores were not significantly different in patients with lower or higher CBFVs (2.73 (0.52) v 2.43 (0.33), NS) in this study. Neither sex nor age were significantly associated with CSF cyto-

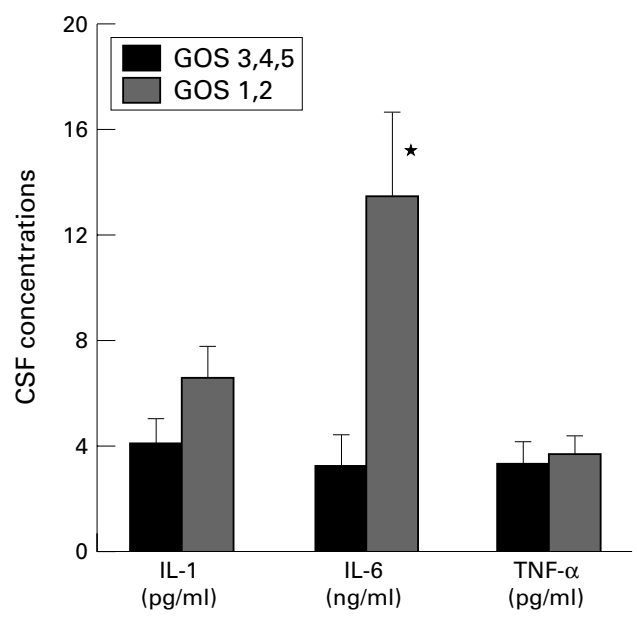

kine concentrations. Thus, age did not correlate with concentrations of IL- $1 \beta(r=0.40, \mathrm{NS})$, IL-6 $(r=0.23, \mathrm{NS})$, or TNF- $\alpha(r=0.14, \mathrm{NS})$. Similarly, concentrations of IL-1 $\beta$ (0.95 (1.45) v 5.03 (0.98) pg/ml, NS), IL-6 (11 763.85 (3134) v 8291.33 (2983) $\mathrm{pg} / \mathrm{ml}, \mathrm{NS}$ ) or TNF- $\alpha$ (3.85 (0.87) v $3.48(0.62) \mathrm{pg} / \mathrm{ml}, \mathrm{NS}$ ) were not significantly different in male or female patients. Moreover, age was not significantly increased in patients with higher CBFVs compared with those with lower CBFVs (57.33 (2.10) v 57.83 (5.04), NS) and mean CBFVs in the middle cerebral artery were not significantly different in male or femal patients (186.08 (16.31) v 325.00 (13.61) cm/s, NS). Finally, age did not correlate with GOS scores $(r=-0.32$, NS) and these scores were not significantly different in male or female patients $(2.31(0.46) v 2.80(0.35), \mathrm{NS})$.

\section{Discussion}

Vasospasms and secondary cerebral ischaemia are the most important complications of SAH. This study showed that the release of IL-1 $\beta$, IL-6, and TNF- $\alpha$ in the subarachnoidal space of patients with SAH is associated with development of increased CBFVs in basal cerebral arteries suggesting a pathophysiological role of excessive subarachnoidal inflammatory host response in haemodynamic complications after SAH.

Secretion of these inflammatory cytokines gradually increased, reaching peak concentrations at days 5-9. Peak concentrations were up to 10000 -fold increased, in the range previously detected in bacterial meningitis. ${ }^{18}$ Interestingly, the haemodynamic abnormalities in recorded basal cerebral arteries of patients with SAH were characterised by a similar subacute temporal profile, only slightly lagging behind the response of the inflammatory cytokines. Release of inflammatory cytokines in the subarachnoidal space was significantly increased in the subpopulation of patients with $\mathrm{SAH}$ with higher CBFVs. These findings are consistent with a recent report of higher IL-6 concentrations in five patients with clinical signs of vasospasms after SAH. ${ }^{16}$ Interestingly, CSF concentrations of IL-6 were significantly increased in patients with SAH with poor clinical outcome, further delineating the clinical relevance of intrathecal cytokine secretion in SAH.

Such pathophysiological importance of compartmentalised cytokine release for development of cerebrovascular complications after SAH could also explain the earlier observations of severe vasospasms in experimental subarachnoidal inflammation ${ }^{19}$ or in bacterial meningitis. ${ }^{18}$ Both conditions are characterised by massive cytokine release ${ }^{18}$ but by an obvious absence of erythrocytes or platelets that are generally considered as key cells in pathophysiology of vasospasms after SAH.

The triggers for the pronounced inflammatory host response in the subarachnoidal space of patients with SAH are unknown. One mechanism could be complement activation, a strong inflammatory stimulus, by osmotically induced disruption of erythrocytes. This is 
suggested by in vitro findings of a complement mediated haemolysis of erythrocytes in subarachnoid blood $\operatorname{clot}^{20}$ or by raised CSF concentrations of complement factors in SAH. ${ }^{21}{ }^{22}$

Subarachnoidal release of inflammatory cytokines could contribute to cerebral vasospasms in several ways: Once released in the subarachnoidal space, these mediators (17-26 $\mathrm{kD})$ could easily access the smooth muscle cells of contiguous basal arteries from their adventitial side as even much larger molecules (for example, horseradish peroxidase, MW $40 \mathrm{kD}$ ) pass from the cysterna magna through the vessel wall to the basal membrane within minutes. ${ }^{23}$ This is possible because the surface of the pial cerebral arteries is, exceptionally, not confined by collagen or fibroblasts but is in direct contact with the nourishing $\mathrm{CSF}^{23} \mathrm{At}$ the smooth vessels, these mediators could directly cause vasospasms although the effects of inflammatory cytokines on vessel tone are still controversial. Whereas some of these mediators (for example, IL-1 $\beta$ ) can act as vasodilators and increase endothelial permeability ${ }^{24-26}$ the cytokine IL-6 which exhibited the strongest response of all cytokines studied in this study, is strongly vasoconstrictive in vitro. ${ }^{16}$ Cytokines may also modulate vessel tone in an indirect manner-for example, by induction of synthesis of vasoconstrictiors such as ET- $1^{27}$ or by induction of adhesion molecule expression responsible for focal leucocyte recruitment. ${ }^{29}$ It has, however, to be noted that further factors may play a part in pathophysiology of cerebral artery narrowing after SAH and multiple therapeutic approaches to cerebrovascular complications after $\mathrm{SAH}$ are currently explored. ${ }^{2}{ }^{30}$

Although we did not find different cytokine concentrations in CSF derived from EVD or by lumbar puncture, effects of the different origin of CSF on these measurements cannot be excluded.

In conclusion, although the clinical and laboratory correlations found cannot prove causal relations, this study supports the hypothesis that an overwhelming inflammatory host response in the subarachnoidal space of patients with SAH plays a central part in pathogenesis of vasospasms and subsequent cerebral ischaemia. As a therapeutic consequence of these findings, we speculate that early subarachnoidal administration of antiinflammatory or anticytokine strategies could prevent haemodynamic complications after SAH.

We are grateful to Mrs M Garcia-Knapp for her secretaria assistance in the preparation of the manuscript.

1 Schievink WI. Intracranial aneurysms. $N$ Engl $f$ Med 1997;336:28-40.

2 Sobey CG, Faraci FM. Subarachnoid haemorrhage: what happens to the cerebral arteries? Clin Exp Pharmacol Physiol 1998;25:867-76.

3 Grosset DG, Straiton J, du Trevou M, et al. Prediction of symptomatic vasospasms after subarachnoid hemorrhage bymptomatic vasospasms after subarachnoid hemorrhage by rapidly increasing transcranial Doppler velocity
ebral blood flow changes. Stroke 1992;23:674-9.
4 Sekhar LN, Wechsler LR, Yonas $\mathrm{H}$, et al. Value of transcranial Doppler examination in the diagnosis of cerebral vaso22:813-21.

5 Seiler RW, Reulen HJ, Huber P, et al. Outcome of aneurysmal subarachnoid hemorrhage in a hospital population: a prospective study including early operation, intravenous nimodipine, and trancranial Doppler ultrasound. Neurosurgery 1988;23:598-604.

6 Sloan MA, Haley EC Jr, Kassell NF, et al. Sensitivity and specificity of transcranial Doppler ultrasonography in the diagnosis of vasospasms following subarachnoidal hemorrhage. Neurology 1989;39:1514-8.

7 Hughes JT, Schianchi PM. Cerebral artery spasm: a histological study at necropsy of the blood vessels in cases of subarachnoidal hemorrhage. $\mathcal{F}$ Neurosurg 1989;48:51525 .

8 Handa Y, Kabuto M, Kobayashi H, et al. The correlation between immunological reaction in the arterial wall and the time course of the development of cerebral vasospasms in a primate mode. Neurosurgery 1991;28:542-9.

9 Minami N, Tani E, Yokota M, et al. Immuno-histochemisty of leukotriene C4 in experimental cerebral vasospasms. Acta Neuropathol 1991;81:401-7.

10 van der Meer JW, Vogels MT, Netea MG, et al. Proinflammatory cytokines and treatment of disease. Ann NY Acad Sci 1998;856:243-51.

11 Kollias G, Douni E, Kassiotis G, et al. On the role of tumor necrosis factor and receptors in models of multiorgan failnecrosis factor and receptors in models of multiorgan fail-
ure, rheumatoid arthritis, multiple sclerosis and inflammaure, rheumatoid arthritis, multiple sclerosis and inflam

12 Mathiesen T, Andersson B, Loftenius A, et al. Increased interleukin-6 levels in cerebrospinal fluid following subarachnoid hemorrhage. $\mathcal{F}$ Neurosurg 1993;78:562-7.

13 Kikuchi T, Okuda Y, Kaito N, et al. Cytokine production in cerebrospinal fluid after subarachnoid hemorrhage. Neurol Res 1995;17:106-8.

14 Mathiesen T, Edner G, Ulfarsson E, et al. Cerebrospinal fluid interleukin-1 receptor antagonist and tumor necrosis factor- $\alpha$ following subarachnoid hemorrhage. 7 Neurosurg 1997;87:215-20

15 Gaetani P, Tartara F, Pignatti P, et al. Cisternal CSF levels of cytokines after subarachnoid hemorrhage. Neurol Res 1998; 20:337-42.

16 Osuka K, Suzuki Y, Tanazawa T, et al. Interleukin-6 and development of vasospasm after subarachnoid haemorrhage. Acta Neurochir (Wien) 1998;140:943-95.

17 Hennerici H, Rautenberg W, Sitzer G, et al. Transcranial Doppler ultrasound for the assessment of intracranial arterial flow velocity. I: examination of technique and normal values. Surg Neurol 1987;27:439-48

18 Fassbender K, Ries S, Schminke U, et al. Inflammatory cytokines in CSF in bacterial meningitis: association with altered blood flow velocities in basal cerebral arteries. 7 Neurol Neurosurg Psychiatry 1996;61:57-61.

19 Peterson JW, Kwun BD, Hackett JD, et al. The role of inflammation in experimental cerebral vasospasm. F Neurosurg 1990;72:767-74.

20 Peterson JW, Kwun BD, Teramura A, et al. Immunological reaction against the aging human subarachnoid erythrocyte. A model for the onset of cerebral vasospasms after subarachnoid hemorrhage. F Neurosurg 1989;71:718-26.

21 Kasuya H, Shimizu T. Activated complement componets $\mathrm{C} 3 \mathrm{a}$ and C4a in cerebrospinal fluid and plasma following subarachnoid hemorrhage. F Neurosurg 1989;71:741-6.

22 Hoshi T, Shimizu T, Kito K, et al. Immunological study of late cerebral vasospasm in subarachnoid hemorrhage. Detection of immunoglobulins, C3, and fibrinogen in cerebral arterial walls by immunoflourescence method. Neurol Med Chir (Tokyo) 1984;24:647-54.

23 Zervas NT, Liszczak TM, Mayberg MR, et al. Cerebrospinal fluid may nourish cerebral vessels through pathways in the adventitia that may be analogous to systemic vasa vasorum. f Neurosurg 1982;56:475-81.

24 McKeating EG, Andrews PJ. Cytokines and adhesion molecules in acute brain injury. Br F Anaesth 1998;80:77-84.

25 Brian JE, Faraci FM, Tumor necrosis factor- $\alpha$-induced dilatation of cerebral arterioles. Stroke 1998;29:509-15.

26 Shibata M, Parfenova H, Zuckerman SL, et al. Interleukin-1 $\beta$ peptides induce cerebral pial arteriolar dilation in peptides induce cerebral pial arteriolar dilation in 50 .

27 Lamas S, Michel T, Collins T, et al. Effects of interferon-y on nitric oxide synthase activity and endothelin-1 production by vascular endothelial cells. f Clin Invest 1992;90; 879-87.

28 Marsden PA, Brenner BM. Transcriptional regulation of endothelin-1 by tumor necrosis factor- $\alpha$. Am $\mathcal{F}$ Physiol Cell Physiol 1992;262;C854-61.

29 Handa Y, Kubota T, Kaneko M, et al. Expression of intercellular adhesion molecule 1 (ICAM-1) on the cerebral artery following subarachnoid haemorrhage in rats. Acta Neurochir (Wien) 1995;132:92-7.

30 Thomas JE, Rosenwasser RH. Reversal of severe vasospams in three patients after aneurysmal subarachnoid in three patients after aneurysmal subarachnoid ventricular sodium nitruprusside in humans. Neurosurgery 1999;44:48-57. 